\title{
The Use of Carbon Nanoparticles for Inkjet-Printed Functional Labels for Smart Packaging
}

\author{
Olha Hrytsenko $\mathbb{D}^{1},{ }^{1}$ Dmytro Hrytsenko ${ }^{\mathbb{D}},{ }^{1}$ Vitaliy Shvalagin $\mathbb{D}^{2}{ }^{2}$ Galyna Grodziuk, ${ }^{2}$ \\ and Mikhail Kompanets $\mathbb{D}^{3}$ \\ ${ }^{1}$ Institute of Publishing and Printing, National Technical University of Ukraine "Igor Sikorsky Kyiv Polytechnic Institute", 1/37 \\ Yangel Str., Kyiv 03056, Ukraine \\ ${ }^{2}$ L.V. Pisarzhevskii Institute of the Physical Chemistry, National Academy of Science of Ukraine, 31 Nauky Ave., Kyiv 03028, Ukraine \\ ${ }^{3}$ L.M. Litvinenko Institute of Physical-Organic Chemistry and Coal Chemistry, 50 Kharkiv Highway, Kyiv 02160, Ukraine
}

Correspondence should be addressed to Olha Hrytsenko; olhasarapulova@gmail.com

Received 18 March 2018; Revised 5 June 2018; Accepted 10 June 2018; Published 2 July 2018

Academic Editor: Domenico Acierno

Copyright $\odot 2018$ Olha Hrytsenko et al. This is an open access article distributed under the Creative Commons Attribution License, which permits unrestricted use, distribution, and reproduction in any medium, provided the original work is properly cited.

Smart packaging functions can be provided by printing functional labels onto packaging materials using inkjet printing and inks with changeable photoluminescence properties. Carbon nanoparticles are considered a perspective fluorescent component of such inks. Ink compositions based on carbon nanoparticles are developed and adapted for inkjet printing on paper packaging materials for producing smart packaging labels. The influence of technological factors of the printing process on the photoluminescence characteristics of the printed images is investigated. The main investigated factors are the concentration of carbon nanoparticles, the relative area of raster elements of a raster field of a tone image, the absorbance and surface smoothness of paper. The resulting parameters are photoluminescence intensity and color. It is found that in case of changes in surface smoothness and absorbance of paper and concentrations of carbon nanoparticles in the ink compositions, the photoluminescence intensity of a printed image changes while its photoluminescence color remains the same. To obtain the highest contrast of tone inkjet-printed images with carbon nanoparticles on papers with any absorbance, the highest concentration of carbon nanoparticles in the ink composition should be used. However, the highest contrast and the highest own photoluminescence intensity of a tone inkjet-printed image with inks with carbon nanoparticles can be achieved only on papers with the lowest absorbance. The most noticeable difference between photoluminescence intensity of printed images on papers with any absorbance can be obtained with the lower concentration of carbon nanoparticles in the ink composition $(10 \mathrm{mg} / \mathrm{mL})$. The optimum concentrations of carbon nanoparticles in the composition are determined: for papers with low absorbance- $-10 \mathrm{mg} / \mathrm{mL}$, and for papers with medium and high absorbance $-25 \mathrm{mg} / \mathrm{mL}$. Analytical dependency is created for photoluminescence intensity of images printed with inkjet printing inks with carbon nanoparticles as a function of the studied technological factors. Some design solutions for photoluminescent labels are suggested.

\section{Introduction}

Smart packaging is becoming more and more popular as a novel solution for customer safety. Its ability to indicate the state of a packaged product as a result of exposure to compounds emerging during food product aging allows smart packaging to supply a consumer actual and reliable information about the safety of a packaged food consumption [1]. Unlike an expiration date, smart packaging takes into consideration the conditions in which the product was stored or transported, the state of a product itself [2, 3], and the state of the internal gas environment of a package $[4,5]$. Smart packaging allows avoiding food poisoning, caused by "fresh" according to the expiration date products, 
which in reality are spoiled due to wrong storage conditions, not to mention tampering with the expiration date and other examples of fraud in supermarket chains, for which the manufacturer often groundlessly takes responsibility.

Smart packaging functions through functional labels on a package surface. These elements react to the predetermined changes of a product (chemical agents that occur in a product as a result of its spoilage, defrosting, and refreezing, as discussed in several studies, for instance $[2,6,7])$ by changing their properties, such as optical, mechanical, or electrical, as is mentioned in [8]. It is obvious that optical changes of properties of the active element are one of the easiest to observe for a customer. Numerous studies demonstrated the fact that nanotechnology offers a wide variety of options for smart packaging solutions, for example, as components for various types of sensors [9, 10], smart labels [11], components of packaging materials for food preservation purposes, as mentioned in [12], for food quality monitoring [13], and even for monitoring the storage conditions or an outside environment of a packaged product, as discussed in [14]. For smart packaging, several studies, for example $[15,16]$, indicate that $\mathrm{ZnO}$ nanoparticles can be used as components for ink compositions for manufacturing functional labels via printing techniques. A more detailed study of the use of $\mathrm{ZnO}$ nanoparticles employing different printing techniques is given in [17]. Besides, the possibility to use $\mathrm{ZnO}$ nanocomposites for printed smart packaging was shown in [18], for antimicrobial packaging, as mentioned in [19], and for the prevention of penetration of UV light, as discussed in [20]. In [21], it was demonstrated that Ag nanoparticles are suitable for various printed smart packaging applications, too. Apart from the abovementioned substances, one of the perspective ones for such purposes is carbon nanoparticles due to their biocompatibility, chemical inertness, availability, and water solubility, as well as the presence of luminescence bands in the visible region of the spectrum. The intelligent function is provided by the reaction of carbon nanoparticles to changes in the composition of a packaged food product by changing the luminescence intensity. Inkjet printing allows to applying carbon nanoparticles in the form of inks on a packaging of a product and to fix them on it. The changes of photoluminescence properties of smart packaging labels can be observed under standard UV lamps for banknote verification at checkout.

The most perspective way to apply carbon nanoparticles onto packaging material is printing. Printing techniques allow high-speed manufacture of low-cost functional labels for smart packaging on an industrial scale. The study [22] demonstrate the possibility of using roll-to-roll gravure printing for smart packaging labels. In [23], the perspectives of screen printing for smart packaging are studied, and some requirements for printing ink compositions with nanoparticles for specific printing techniques are discussed. Among other printing techniques, inkjet printing is already used to print manufacture date, expiration date, and other technical data onto a package about the same time as the product is being packed. The use of inkjet printing for application of a functional label on smart packaging is also possible, as argued in [21], and inkjet printing allows to avoid costly reequipment of an enterprise for the production of such type of packaging. As mentioned in [21], apart from such functional labels, printed images with fluorescent properties can be used for security printing purposes. This is also a prospective application of inkjet printing inks based on carbon nanoparticles.

Therefore, in order to enable the use of carbon nanoparticles for inkjet-printed functional labels for smart packaging, the printing inks based on carbon nanoparticles should be created and their application to packaging materials should be studied considering the influence of technological factors of the printing process on photoluminescence properties of the obtained printed images. The aim of this study is to determine the effect of process parameters of applying ink compositions containing carbon nanoparticles onto paper packaging materials by inkjet printing on the photoluminescent properties of the obtained printed images.

\section{Materials and Methods}

2.1. Carbon Nanoparticle Preparation. Nanoparticles of carbon were prepared by thermal treatment using a solid-state reaction method of a mixture of dry citric acid and thiourea with a molar ratio of the components of $1: 3$ respectfully at $200^{\circ} \mathrm{C}$ for $30 \mathrm{~min}[24,25]$. Reducing or increasing the time of thermal treatment in our conditions leads to a decrease in the luminescence intensity. In case of a reduction in the time of thermal treatment, this may be due to a small number of formed carbon nanoparticles. With an increase in the time of thermal treatment, obviously, there is a more complete carbonization with the formation of large-sized carbon particles, which have a lower luminescence intensity. Before thermal treatment, the precursors were thoroughly rubbed in an agate mortar. After the heat treatment, the fried material was dissolved in water and a nonluminescent precipitate was separated by centrifugation at $3000 \mathrm{rpm}$ for 10 minutes. After centrifugation, a transparent colloid of yellow color with a slight opalescence was obtained. The concentration of the obtained carbon nanoparticles was about $50 \mathrm{mg} / \mathrm{mL}$, measured by weighing the dry residue after evaporation of the solvent from a certain volume of colloid. In contrast to the method described in the literature [24], in this work, the thermal treatment was not hydrothermal in the autoclave, but it was carried out by the heat treatment of the dry mixture (i.e., solidstate reaction method), which significantly reduces the time of the thermal treatment, eliminates the use of high pressures, and allows to scale up the synthesis. In this paper, the authors did not study the conditions for varying the size of carbon nanoparticles, since the main focus was on obtaining a material with the greatest possible luminescence intensity in our conditions.

2.2. Inkjet Printing Ink Preparation. The inkjet printing ink was created by diluting the obtained colloidal solution of carbon nanoparticles with distilled water to obtain the following concentrations of carbon nanoparticles: $5 \mathrm{mg} / \mathrm{mL}$, $10 \mathrm{mg} / \mathrm{mL}$, and $25 \mathrm{mg} / \mathrm{mL}$. The initial solution of carbon nanoparticles has the viscosity compatible with inkjet printing 
equipment and technology; these solutions are suitable for the stable printing process on paper materials (i.e., absorbing materials).

2.3. Inkjet Printing Set-Up. The printed images were obtained using inkjet printing with the developed ink compositions based on carbon nanoparticles onto paper substrates. The test form was designed consisting of raster areas $2 \mathrm{~cm} \times 2 \mathrm{~cm}$ with different relative areas of raster elements $(20 \%, 40 \%$, $60 \%, 80 \%$, and $100 \%$ ). The paper for inkjet printing was selected on the basis of its low content of optical brightness agents (OBAs) in order to avoid interference of own photoluminescence of a substrate with the photoluminescence of a printed image because intensive photoluminescence of a paper often can quench the photoluminescence of a printed image completely.

The papers have different surface smoothness and absorbance. The paper smoothness is measured by Becca and is to measure the time of passage of a certain volume of air in the vacuum chamber between the sample surface and a polished glass plate surface in certain conditions.

The influence of paper absorbance on photoluminescence properties of inkjet-printed images was also investigated. The absorbance ability of the paper is characterized by its degree of sizing. The degree of the sizing of the paper can be defined by a stroke technique. According to this technique, the standard ink is applied onto a substrate (in this case, paper) by drawing pens graded in a series of strokes of an equal length of $75 \mathrm{~mm}$ and a width that is growing (i.e. $0.25 \mathrm{~mm}, 0.5 \mathrm{~mm}, 0.75 \mathrm{~mm}, 1.0 \mathrm{~mm}$, and $1.25 \mathrm{~mm}$ ). The width of the stroke that has the largest width, which is not blurred on the substrate and does not pass through it at the same time, indicates the degree of sizing of the substrate in $\mathrm{mm}$.

2.4. Investigated Technological Factors. Among all the technological factors which can be controlled during the printing process, the following factors were considered to have the most significant influence on photoluminescence properties of the obtained images; so in this study, they varied as follows: (1) the content of printing ink varied depending on the concentration of an initial carbon nanoparticle solution in the ink, namely, $5 \mathrm{mg} / \mathrm{mL}, 10 \mathrm{mg} / \mathrm{mL}$, and $25 \mathrm{mg} / \mathrm{mL}$; (2) the relative area of raster elements was the following: $20 \%$, $40 \%, 60 \%, 80 \%$, and $100 \%$; (3) the absorbance of the paper was the following: relatively low for paper A (with the degree of sizing of $2.0 \mathrm{~mm}$ ), medium for paper B (with the degree of sizing of $1.25 \mathrm{~mm}$ ), and the highest for paper $\mathrm{K}$ (with the degree of sizing of $0.75 \mathrm{~mm}$ ); (4) the surface smoothness of the papers was the following: the highest for paper A $(125 \mathrm{sec})$, medium for paper B $(95 \mathrm{sec})$, and the lowest for paper $\mathrm{K}(65 \mathrm{sec})$.

The main investigated parameters of photoluminescence of the printed images obtained using the inks with carbon nanoparticles are photoluminescence intensity and photoluminescence color. The photoluminescence color is defined by the interrelation of the positions and peak heights of photoluminescence bands.
2.5. Equipment Used. An inkjet printer Epson Stylus SX 4300 with 600 dpi resolution was used to apply the developed inkjet printing ink onto paper substrates. Optical density spectra were recorded with a spectrophotometer Analytik Jena Specord 210. Photoluminescence spectra were recorded with a photoluminescence spectrometer Perkin-Elmer LS55. Transmission electron microscopy (TEM) data were obtained with a Selmi PEM-125K (Ukraine) with an accelerating voltage of $100 \mathrm{kV}$.

\section{Results and Discussion}

3.1. Characterization of the Obtained Carbon Nanoparticles. According to TEM microphotographs (see Figure 1), the size of the obtained carbon nanoparticles is in the nanometer range and is about $30 \mathrm{~nm}$.

The edge of the absorption band of the resulting solution of carbon nanoparticles is at $450 \mathrm{~nm}$ (Figure 2, curve 1). At the same time, there is a slight absorption in the longer-wave region $(450-700 \mathrm{~nm})$, which may be due to partial scattering of light by a concentrated colloid of carbon nanoparticles. When diluting the initial colloid 10 times, a clear maximum at $300 \mathrm{~nm}$ is observed in the absorption spectrum (Figure 2, curve 2). This peak may be due to $n \rightarrow \pi^{*}$ transition of the $\mathrm{C}=\mathrm{O}$ bond [26]. It should be noted that for the material obtained by hydrothermal treatment of citric acid and thiourea, the indicated maximum is shifted to a longer-wave region and is at $335 \mathrm{~nm}$ [13]. Also, in our case, there are no long-wave absorption bands at $422 \mathrm{~nm}, 550 \mathrm{~nm}$, and $595 \mathrm{~nm}$ [13], which may indicate the weak doping of carbon nanoparticles with sulfur. The indicated changes are obviously related to the heat treatment in the open crucible, which can lead to the removal of sulfur in the form of volatile oxides.

As shown in Figure 2, in order to obtain the maximum intensity of photoluminescence solution of carbon nanoparticles, it is expedient to excite photoluminescence with UV light with a wavelength $\lambda_{\text {excit }}<400 \mathrm{~nm}$. Actually, as can be seen from the photoluminescence excitation spectrum (Figure 3, curve 1), the maximum luminescence intensity of the obtained carbon nanoparticles can be achieved by excitation with light with $\lambda=370 \mathrm{~nm}$ (maximum on curve 1 , Figure 3).

Besides, as can be seen from Figure 3, the photoluminescence spectrum of the solution of carbon nanoparticles when excited with light with $\lambda_{\text {excit }}=370 \mathrm{~nm}$ is a characterized wide band of emission in the range $400-700 \mathrm{~nm}$ with a clearly expressed peak at $\lambda=460 \mathrm{~nm}$ (Figure 3 , curve 2). A change in the excitation wavelength, as can be seen from Figure 3 curve 1 , leads to a significant decrease in the luminescence intensity. At the same time, in our case, the form of the emission spectrum (which determines the color of radiation) practically does not change.

3.2. Characterization of the Obtained Printed Images. Figure 4 shows the photo of the obtained inkjet-printed images under UV light and daylight for three types of papers which differ by their absorbance: paper A-the lowest absorbance and the highest surface smoothness, paper B-the 


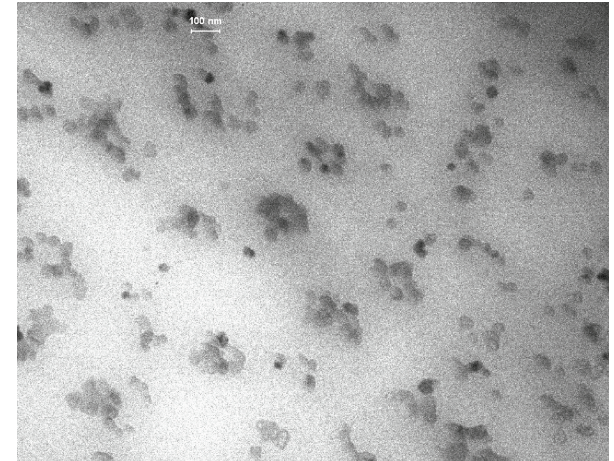

FIGURE 1: TEM photograph of the obtained carbon nanoparticles.

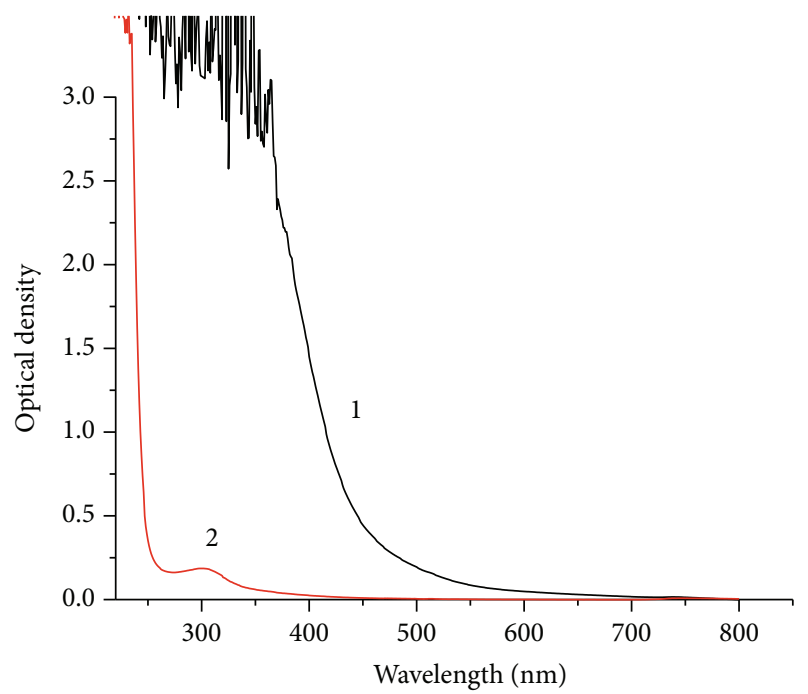

Figure 2: The absorption spectrum of a solution of carbon nanoparticles with the concentrations of $50 \mathrm{mg} / \mathrm{mL}(1)$ and $5 \mathrm{mg} /$ $\mathrm{mL}(2) . l=10 \mathrm{~mm}$.

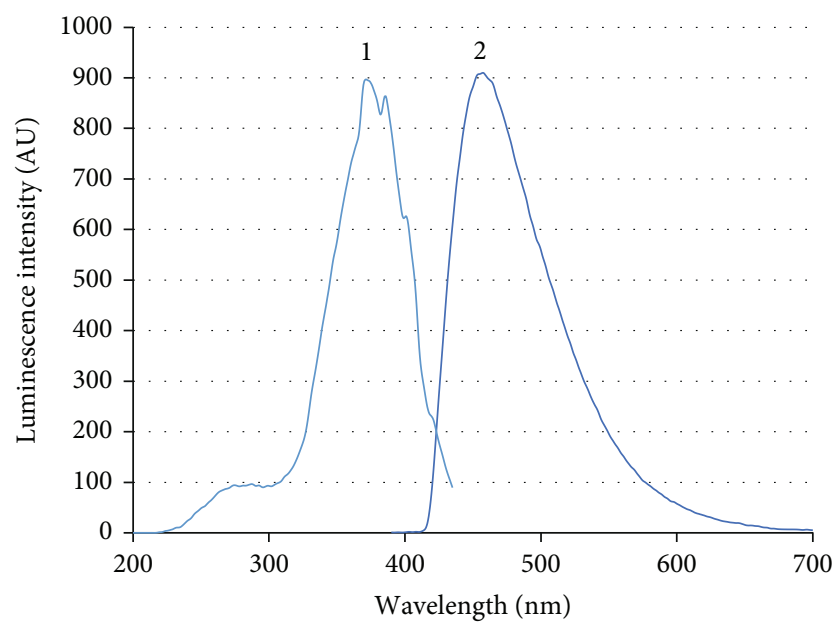

Figure 3: The photoluminescence excitation spectrum (1) and photoluminescence spectrum (2) of the solution of carbon nanoparticles; $\lambda_{\text {excit }}=370 \mathrm{~nm}$. average absorbance and the average surface smoothness, and paper $\mathrm{K}$-the highest absorbance and the lowest surface smoothness among the investigated non-OBA papers.

Figure 5 shows the photoluminescence spectra of raster fields with the following relative area of raster elements: $0 \%$ (photoluminescence of paper), $10,20, \ldots, 100 \%$ for paper A; the concentration of the fluorescent component (carbon nanoparticles) in the ink composition is $25 \mathrm{mg} /$ $\mathrm{mL}$. Such photoluminescence spectra are typical for all the investigated papers. They differ only in the height (intensity) of photoluminescence peak at $\lambda=440 \mathrm{~nm}$. It is determined that the photoluminescence peak position is not changed for the studied paper types and concentrations of carbon nanoparticles. This means that a change of photoluminescence intensity happens without any change in photoluminescence color.

Figure 6 shows the gradation curves-the summary of the heights of photoluminescence spectra peaks for raster fields with the following relative area of raster elements: $0 \%$ (photoluminescence of paper) $, 10,20, \ldots, 100 \%$, for inks with the concentrations of carbon nanoparticles of $5 \mathrm{mg} / \mathrm{mL}, 10 \mathrm{mg} / \mathrm{mL}$, and $25 \mathrm{mg} / \mathrm{mL}$ applied onto nonOBA papers.

As shown in Figure 6, the gradation curves for different types of paper have different shapes. From the gradation curves in Figure 6, the contrast can be determined (the difference between the lightest and the darkest areas of the image) for the images printed on the studied papers (Figure 5).

As shown in Figure 7, the highest contrast of a tone image can be obtained with the use of paper A (with the lowest absorbance among the studied types of papers) and at the concentration of carbon nanoparticles in the ink of $25 \mathrm{mg} /$ $\mathrm{mL}$. As the concentration of carbon nanoparticles in the printing ink on paper A decreases, the contrast decreases less significantly in comparison with papers B and C (the papers with higher absorbance). In other words, the increase in the concentration of carbon nanoparticles leads to the increase of the contrast of a printed image, and the effect is more significant for papers with higher absorbance. In general, the use of the highest of the studied concentrations of carbon nanoparticles $(25 \mathrm{mg} / \mathrm{mL})$ in the ink composition makes it possible to obtain the highest contrast of a tone image on papers with the same absorbance.

Since own photoluminescence of the paper causes a significant impact on the photoluminescence intensity of the printed images, the photoluminescence intensities of papers were subtracted from the photoluminescence intensities of the printed images. The resulting data are presented in Figure 8.

As shown in Figure 8, at the same concentration of carbon nanoparticles, the shapes of the gradation curves on different types of paper are different: the paper with the lowest absorbance (paper A) allows to obtain the highest intensity of own photoluminescence of printed images (excluding photoluminescence of paper). In this case, the lower the concentration of carbon nanoparticles in the composition, the more significant the difference is between the photoluminescence intensities of printed images on papers with low 

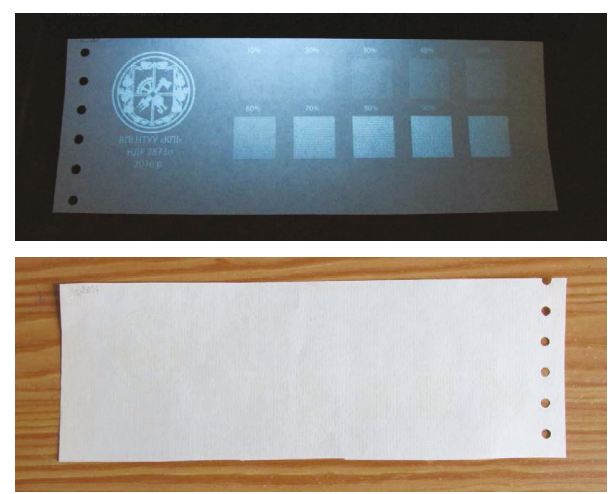

(a)
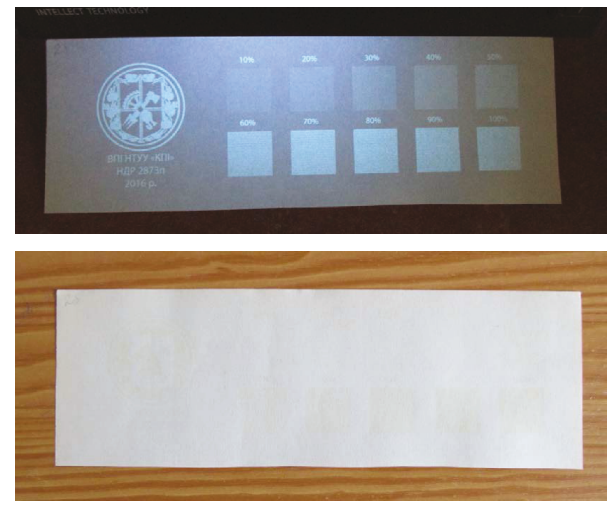

(b)
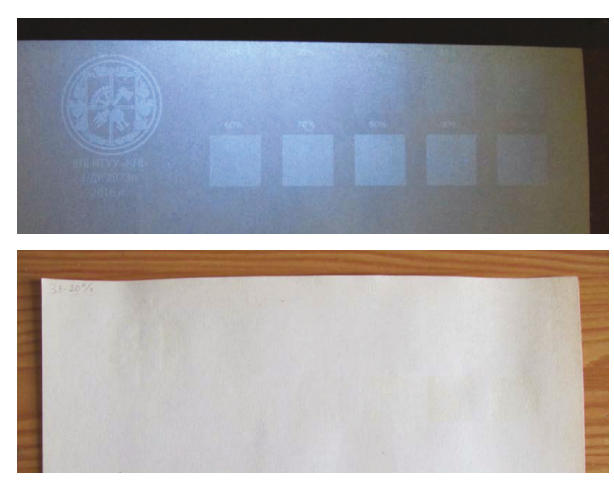

(c)

FIgURE 4: The photo of the obtained inkjet-printed images under UV light and daylight for three types of papers which differ by their absorbance: (a) paper A, (b) paper B, and (c) paper $\mathrm{K}$. Concentration of carbon nanoparticles in the ink; $25 \mathrm{mg} / \mathrm{mL}$. Currency detector Pro 7 Intellect Technology (UV lamp output is $7 \mathrm{~W})$.

absorbance (paper A) and the papers with higher absorbance (paper B and paper C).

For a more detailed study of this phenomenon, there were built dependencies of own photoluminescence intensity of the printed images on the concentration of carbon nanoparticles for the set of relative areas of raster elements of raster fields $(20,50,70$, and $100 \%)$ for each of the studied papers alone (Figure 9).

Thus, it was found that for papers with low absorbance (paper A), the optimum concentration of carbon
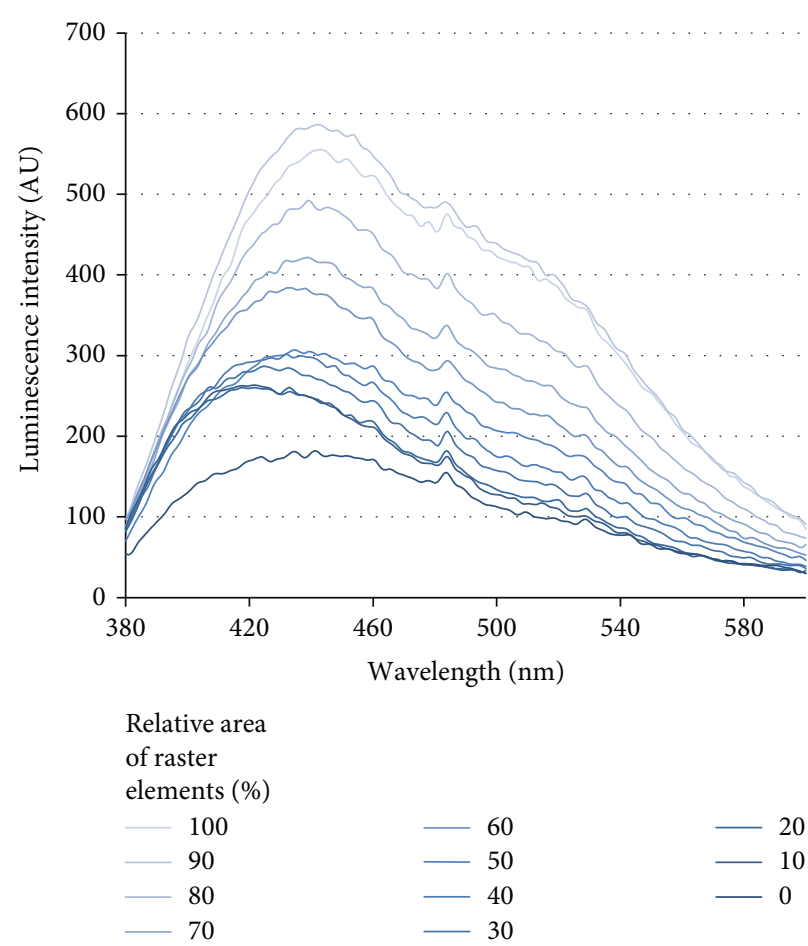

Figure 5: The photoluminescence spectra of raster fields with a relative area of raster elements: 0 (photoluminescence of paper), $10,20, \ldots, 100 \%$ for paper A. $\lambda_{\text {excit }}=370 \mathrm{~nm}$; $[\mathrm{C}]=25 \mathrm{mg} / \mathrm{mL}$.

nanoparticles in the ink composition is $10 \mathrm{mg} / \mathrm{mL}$. The use of larger concentrations is not recommended because with the increase of the concentration of carbon nanoparticles in the composition up to $25 \mathrm{mg} / \mathrm{mL}$ at the low relative areas of raster elements $(20 \%, 50 \%)$, own photoluminescence intensity of printed images decreases by $44.1 \%$ and $33.7 \%$, respectively. At the high relative areas of raster elements (70\%, $100 \%$ ), it reduces slightly (by 5.7\%) and remains unchanged accordingly (Figure 9(a)).

For papers with average absorbance (paper B), the optimum concentration of carbon nanoparticles in the ink composition is $25 \mathrm{mg} / \mathrm{mL}$, since the use of high concentrations of carbon nanoparticles in the ink composition allows to significantly increase the own photoluminescence intensity of printed images at high relative areas of raster elements $(70 \%, 100 \%)$ by $85.9 \%$ and $58.0 \%$, respectively. However, the own photoluminescence intensity of printed images at low relative areas of raster elements $(20 \%, 50 \%)$ rises less significantly (by $47.1 \%$ ) or remains unchanged, respectively (Figure 9(b)).

For papers with high absorbance (paper K), the optimum concentration of carbon nanoparticles in the ink composition is $25 \mathrm{mg} / \mathrm{mL}$, since the use of high concentrations of carbon nanoparticles in the ink composition can drastically increase the own photoluminescence intensity of printed images at high relative areas of raster elements $(70 \%, 100 \%)$ by $128.1 \%$ and $217.9 \%$, respectively. However, the own photoluminescence intensity of printed images at low relative areas of raster elements $(20 \%, 50 \%)$ slightly reduces (by $51 \%$ ) or increases less significantly (by 52.6\%) (Figure 9(c)). 

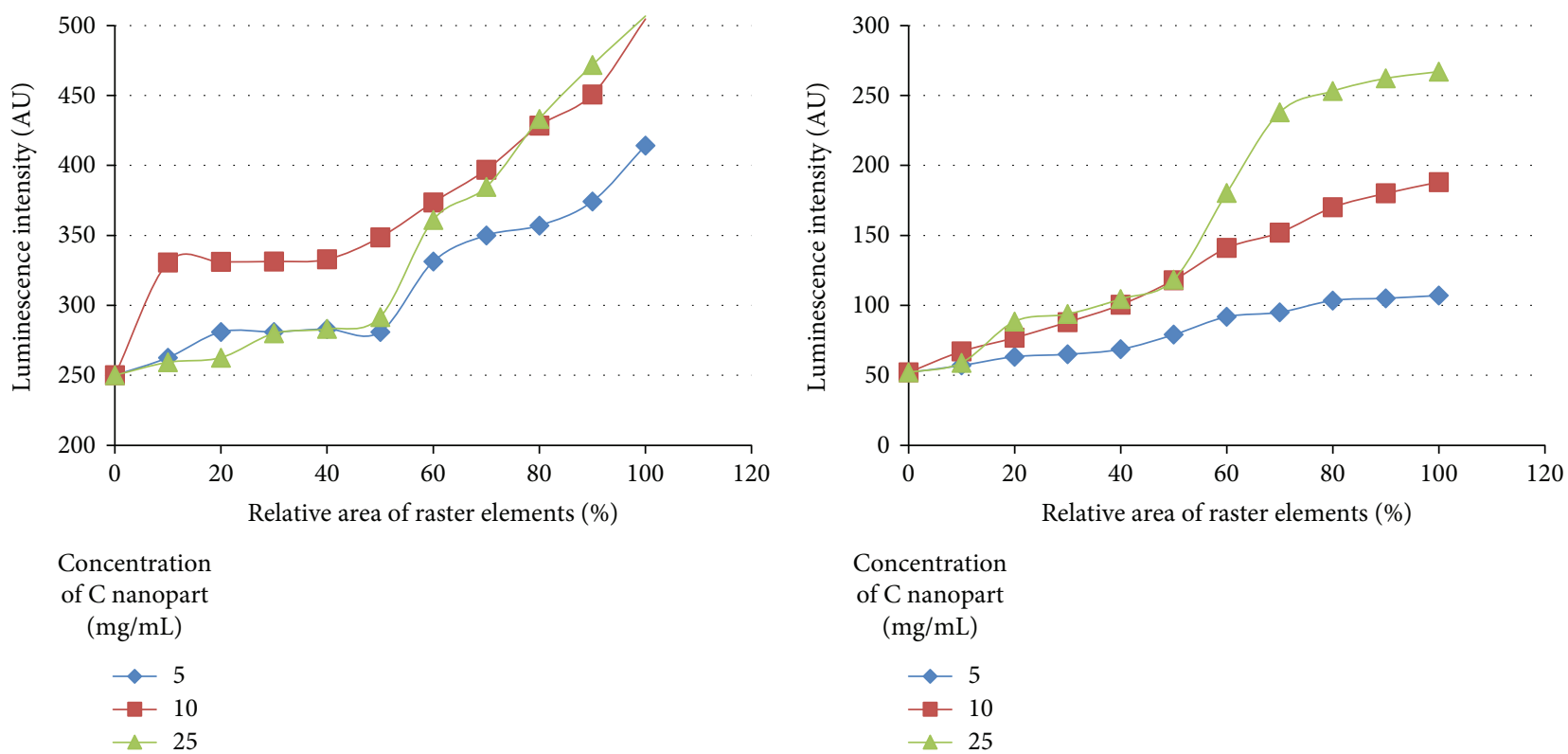

(a)

(b)

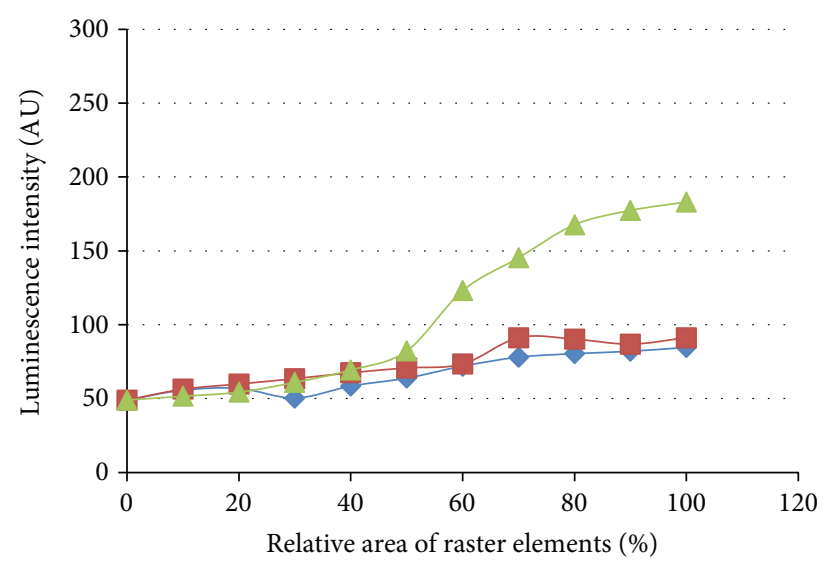

$$
\begin{aligned}
& \text { Concentration } \\
& \text { of C nanopart } \\
& (\mathrm{mg} / \mathrm{mL}) \\
& -5 \\
& -10 \\
& -25
\end{aligned}
$$

(c)

FIgURE 6: The dependencies of photoluminescence intensity of inkjet-printed images at $\lambda=440 \mathrm{~nm}$ on the relative area of raster elements of raster fields: (a) paper A (low absorbance), (b) paper B (average absorbance), and (c) paper K (the highest absorbance among the studied papers).

3.3. Mathematical Model. A full factorial experiment was conducted, and a mathematical model was created to obtain the analytical dependence of photoluminescence intensity of printed images $I=f(c, R, s)$, which takes into account the impact of the following factors on the photoluminescence intensity of the printed images with inks based on carbon nanoparticles for inkjet printing: $c$ is the concentration of carbon nanoparticles, $\mathrm{mg} / \mathrm{mL} ; R$ is the relative area of raster elements of raster field, $\%$; $s$ is the surface smoothness of a paper, sec. The surface smoothness of the investigated papers varied within 65$125 \mathrm{sec}$.

The equation of a mathematical model in normalized coordinates is

$$
\begin{aligned}
y\left(\tilde{x}_{1}, \tilde{x}_{2}, \tilde{x}_{3}\right)= & 22,94 \tilde{x}_{1}+69,9325 \tilde{x}_{2}+133,4925 \tilde{x}_{3} \\
& +24,8025 \tilde{x}_{1} \cdot \tilde{x}_{2}-0,6525 \tilde{x}_{1} \cdot \tilde{x}_{3}+29,81 \tilde{x}_{2} \\
& \cdot \tilde{x}_{3}-0,85 \tilde{x}_{1} \cdot \tilde{x}_{2} \cdot \tilde{x}_{3}+227,205 .
\end{aligned}
$$




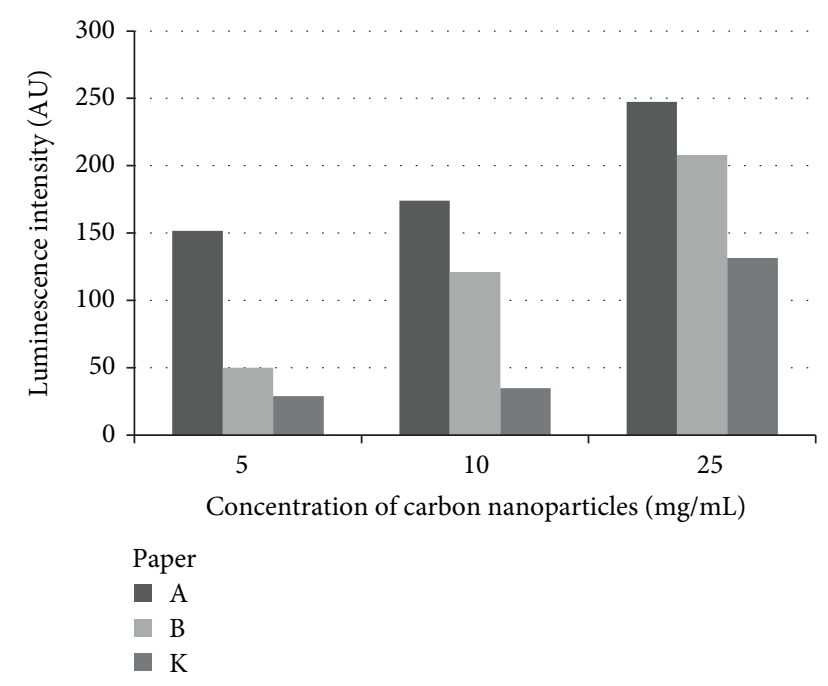

FIgURE 7: Contrast of reproduction of gradations with photoluminescent printing inks for papers $\mathrm{A}, \mathrm{B}$, and $\mathrm{K}$ at the concentration of carbon nanoparticles in the ink compositions of $5 \mathrm{mg} / \mathrm{mL}, 10 \mathrm{mg} / \mathrm{mL}$, and $25 \mathrm{mg} / \mathrm{mL}$.

The equation of a mathematical model in nonnormalized (native) coordinates is

$$
\begin{aligned}
I= & -1.00114 c-1,46,016 R+3,103,403 s \\
& +0.061098 c \cdot R-0.001288 c \cdot s \\
& +0,023026 R \cdot s-6,2963 \cdot 10^{-5} c \cdot R \cdot s-135,119,
\end{aligned}
$$

where $c$ is the concentration of carbon nanoparticles, $\mathrm{mg} / \mathrm{mL}$; $R$ is the relative area of raster elements of raster field, \%; and $s$ is the surface smoothness of paper, sec.

3.4. The Effects of the Studied Technological Factors. To sum up, the effects were studied of technological factors of the inkjet printing technique on photoluminescence properties of printed images with carbon nanoparticles. Based on the calculated analytical dependence (in normalized coordinates) of photoluminescence intensity of printed images on the concentration of carbon nanoparticles, the relative area of raster elements of raster fields, and the surface smoothness of paper, it was stipulated that

(1) The surface smoothness of the paper has the greatest influence on the photoluminescence intensity of inkjet-printed images with carbon nanoparticles in the ink composition.

(2) The relative area of raster elements of raster fields has less influence on the photoluminescence intensity of inkjet-printed images with carbon nanoparticles in the ink composition.

(3) The concentration of carbon nanoparticles in the ink composition has relatively the least impact on the photoluminescence intensity of inkjet-printed images. However, this is due to the fact that it is recommended to choose the average concentration of the photoluminescent component (carbon nanoparticles) in the ink composition $(10 \mathrm{mg} / \mathrm{mL})$ because higher concentration leads to an insignificant increase in the photoluminescence intensity of the resulting printed image.

The comparison of the impact values of the studied technological factors on the luminescence intensity of the printed labels was conducted in order to determine which technological factors are the most effective to be varied to increase or decrease the output luminescence intensity of the printed labels.

Surface smoothness of the paper has the greatest effect on the photoluminescence intensity due to the fact that the surface of a more rough paper is less covered with ink, ink is more absorbed between fibers of the paper. At the same time, in irradiation with UV radiation of the image on a smooth paper, it physically receives more UV light directed vertically at the image, while the roughness of paper causes some optical quenching in case of irradiation of a label with UV light as well as fluorescent emission returning to an observer.

The relative area of a raster element also causes the influence on the luminescence intensity, since it expresses the physical amount of ink (and hence the amount of luminescent substance) distributed in the area of a printed image. However, when comparing the impact values of the above technological factors, it was experimentally found that surface smoothness of the paper has a relatively greater impact value in percentage terms than the relative area of a raster element.

When comparing the impact values of the abovementioned technological factors, it has been experimentally found that the concentration of carbon nanoparticles has a relatively small influence on the luminescence intensity of the samples in percentage terms than the surface smoothness of paper and the area of the raster element of the raster field. However, this influence is still significant and must be taken into account (see Figures 7, 9(b) and 9(c)).

In addition to providing advanced functionality to food packaging, printed photoluminescent labels can perform a finishing function. Combining printed with ink photoluminescent ink elements of an image with the elements of those printed by traditional printing inks, several options of design solutions can be reproduced:

(1) An invisible in daylight image printed solely with photoluminescent inks (see Figure 3)

(2) A combined image-partly visible in daylight image containing the elements reproduced with photoluminescent inks and visible only when irradiated with UV light

A variation of the latest option is a smart packaging label with the standard for comparison. Such label is divided into two parts, one of which is in contact with a packaged product and changes its optical characteristics in response to changes in the state of a packaged product. The second part is not in contact with the product and do not change its optical characteristics and serves as a benchmark for comparison. 


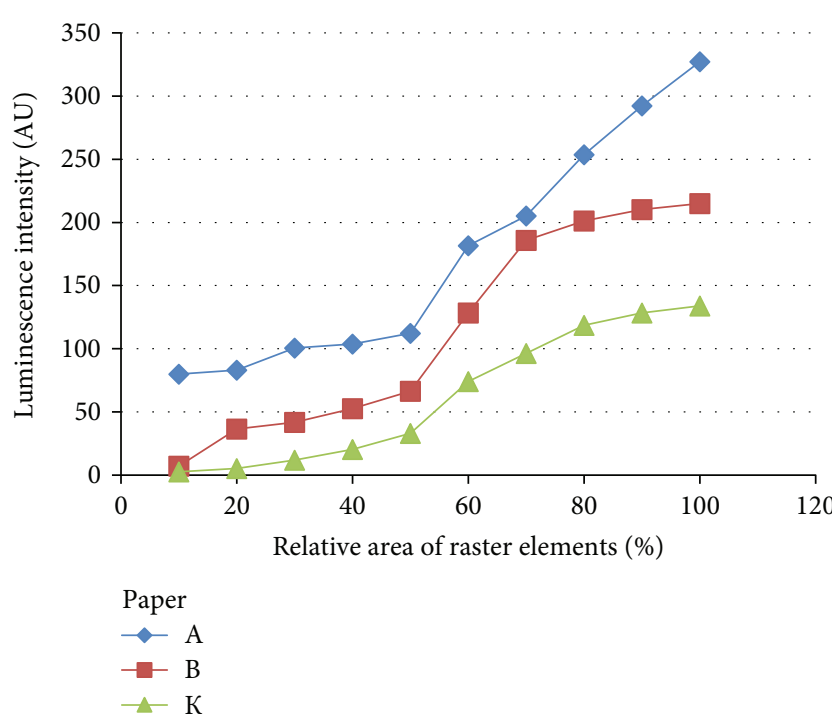

(a)

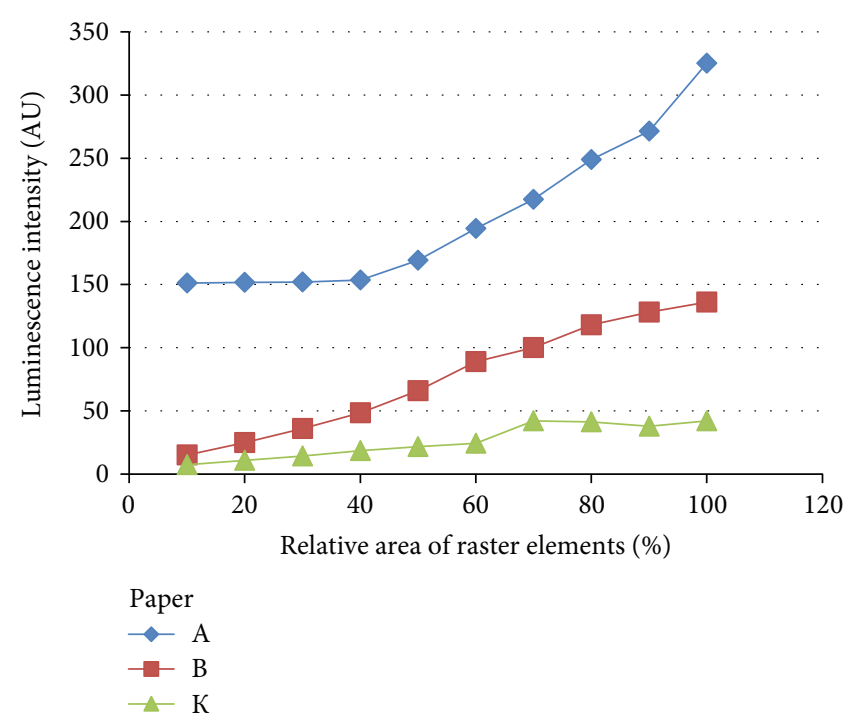

(b)

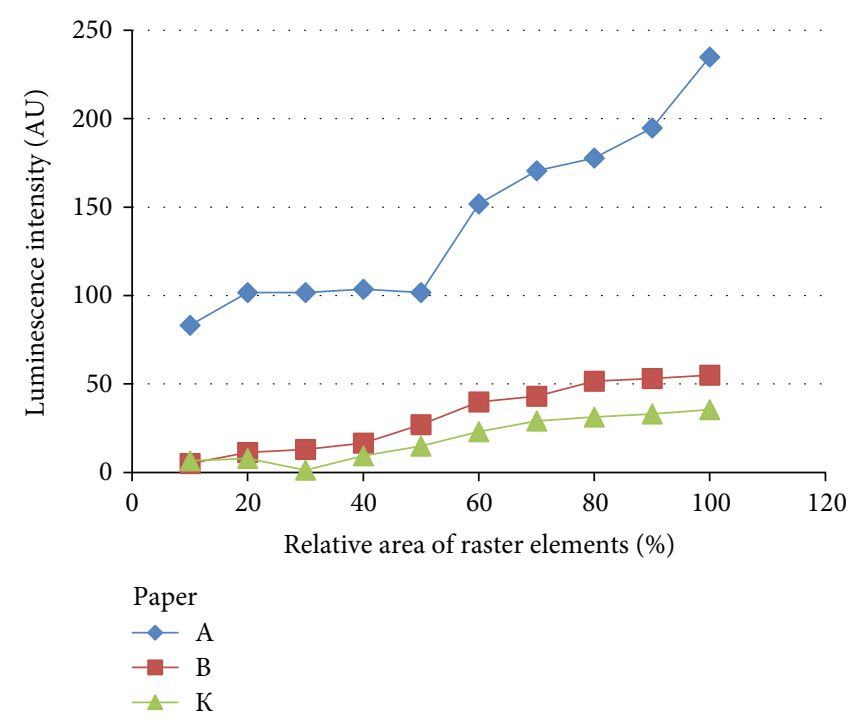

(c)

FIGURE 8: The dependencies of intensities of own photoluminescence (photoluminescence without paper) of the inkjet-printed images at $\lambda=440 \mathrm{~nm}$ on relative area of raster elements of raster fields on paper A (low absorbance), paper B (average absorbance), and paper K (the highest absorbance among the studied papers). The concentrations of carbon nanoparticles in the ink composition are the following: (a) $25 \mathrm{mg} / \mathrm{mL}$, (b) $10 \mathrm{mg} / \mathrm{mL}$, and (c) $5 \mathrm{mg} / \mathrm{mL}$.

\section{Conclusions}

In this research, there are investigated parameters of the process of inkjet printing with ink compositions containing carbon nanoparticles on photoluminescence characteristics of the obtained printed images. It was found that the position of photoluminescence peak of the printed images does not change for the studied paper types and concentrations of carbon nanoparticles. In this case, only the photoluminescence peak height changes. It means that the photoluminescence intensity of these cases changes while the photoluminescence color remains the same. The highest contrast of tone images on papers with any absorbance can be obtained using the highest studied concentration of carbon nanoparticles $(25 \mathrm{mg} / \mathrm{mL})$ in the ink composition. At any concentration of carbon nanoparticles in the ink composition $(5 \mathrm{mg} / \mathrm{mL}, 10 \mathrm{mg} / \mathrm{mL}$, or $25 \mathrm{mg} / \mathrm{mL})$, the highest contrast of tone image can be achieved by the use of papers with the lowest absorbance. Papers with the lowest absorbance also allow obtaining the highest own photoluminescence intensity of printed impressions (excluding photoluminescence of paper). The lower the concentration of carbon nanoparticles in the ink composition is, the more noticeable the difference between photoluminescence intensities of printed images on paper with low absorbance and papers with higher absorbance. The optimum 


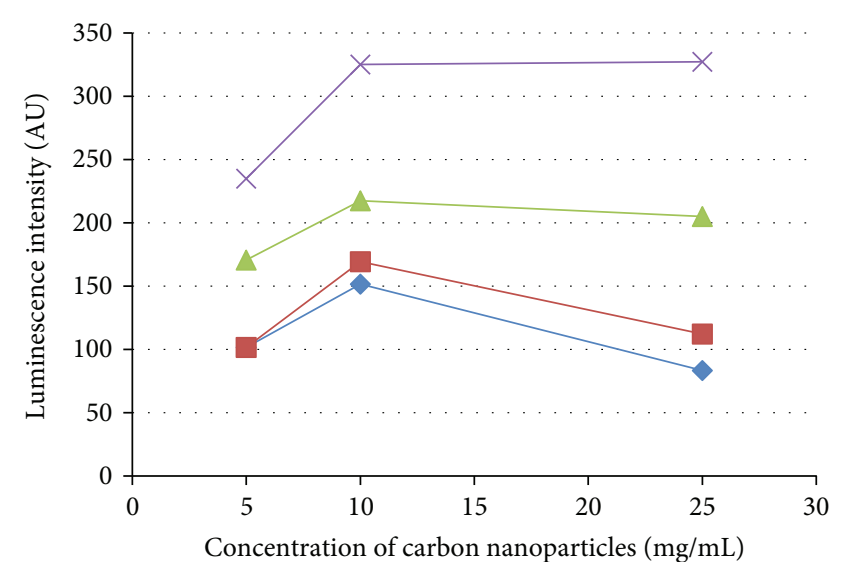

Relative area

of raster

elements (\%)

$\rightarrow-20$

$-50$

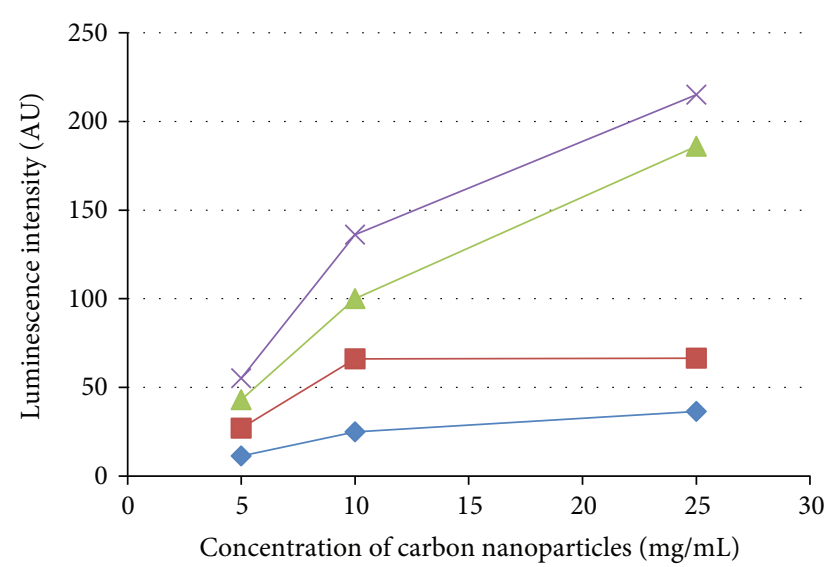

Relative area

of raster

elements (\%)

$\rightarrow-20$

$=50$

$-70$

$\times 100$

(a)

(b)

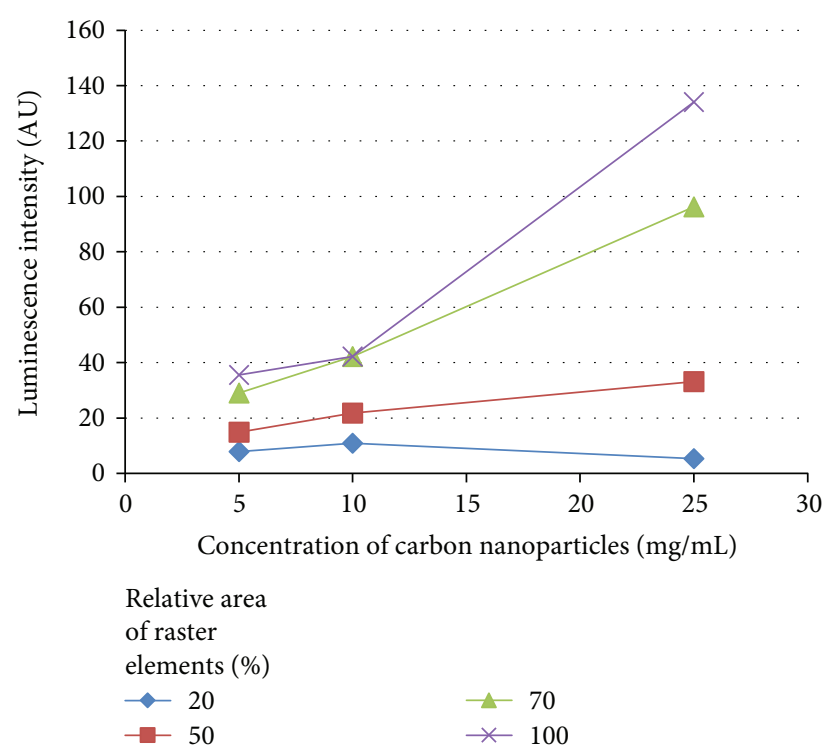

(c)

FIgURE 9: The dependencies of the own photoluminescence intensity of the printed images at $\lambda=440 \mathrm{~nm}$ on the concentration of carbon nanoparticles for relative area of raster elements of raster fields of 20,50, 70, and 100\% for the following types of papers: (a) paper A (low absorbance), (b) paper B (average absorbance), and (c) paper K (the highest absorbance among the studied papers).

concentrations of carbon nanoparticles in the composition are determined: for papers with low absorbance- $10 \mathrm{mg} /$ $\mathrm{mL}$, and for papers with medium and high absorbance- $-25 \mathrm{mg} / \mathrm{mL}$. Analytical dependence is calculated for the photoluminescence intensity of images printed with inkjet printing inks with carbon nanoparticles as a function of the concentration of carbon nanoparticles, the relative area of raster elements of a raster field, and the surface smoothness of paper. A few design solutions for photoluminescent labels are suggested for them to perform not only informative but also finishing features, combined with the function of protection against forgery. This is achieved by combining parts of an image printed visible in daylight inks and inks with carbon nanoparticles which are visible only under UV light.

\section{Data Availability}

The data are available on request. Olha Hrytsenko should be contacted via email at olhasarapulova@gmail.com.

\section{Conflicts of Interest}

The authors declare that there is no conflict of interest regarding the publication of this paper. 


\section{Acknowledgments}

Publications are based on the research provided by the grant support of the State Fund for Fundamental Research of Ukraine (Project N F64/10-2016).

\section{References}

[1] B. Kuswandi, Y. Wicaksono, A. A. Jayus, L. Y. Heng, and M. Ahmad, "Smart packaging: sensors for monitoring of food quality and safety," Sensing and Instrumentation for Food Quality and Safety, vol. 5, no. 3-4, pp. 137-146, 2011.

[2] J. Kerry and P. Butler, Smart Packaging Technologies for Fast Moving Consumer Goods, John Wiley, 2008.

[3] V. Kumar, P. Guleria, and S. K. Mehta, "Nanosensors for food quality and safety assessment," Environmental Chemistry Letters, vol. 15, no. 2, pp. 165-177, 2017.

[4] P. Puligundla, J. Jung, and S. Ko, "Carbon dioxide sensors for intelligent food packaging applications," Food Control, vol. 25, no. 1, pp. 328-333, 2012.

[5] J. W. Rhim, H. M. Park, and C. S. Ha, "Bio-nanocomposites for food packaging applications," Progress in Polymer Science, vol. 38, no. 10-11, pp. 1629-1652, 2013.

[6] A. Pacquit, J. Frisby, D. Diamond et al., "Development of a smart packaging for the monitoring of fish spoilage," Food Chemistry, vol. 102, no. 2, pp. 466-470, 2007.

[7] K. Ramachandraiah, S. G. Han, and K. B. Chin, "Nanotechnology in meat processing and packaging: potential applications-a review," Asian-Australasian Journal of Animal Sciences, vol. 28, no. 2, pp. 290-302, 2015.

[8] G. Fuertes, I. Soto, R. Carrasco, M. Vargas, J. Sabattin, and C. Lagos, "Intelligent packaging systems: sensors and nanosensors to monitor food quality and safety," Journal of Sensors, vol. 2016, Article ID 4046061, 8 pages, 2016.

[9] J. Momin, C. Jayakumar, and J. Prajapati, "Potential of nanotechnology in functional foods," Emirates Journal of Food and Agriculture, vol. 25, no. 1, p. 10, 2013.

[10] Y. Wyser, M. Adams, M. Avella et al., "Outlook and challenges of nanotechnologies for food packaging," Packaging Technology and Science, vol. 29, no. 12, pp. 615-648, 2016.

[11] S. Neethirajan and D. S. Jayas, "Nanotechnology for the food and bioprocessing industries," Food and Bioprocess Technology, vol. 4, no. 1, pp. 39-47, 2011.

[12] T. V. Duncan, "Applications of nanotechnology in food packaging and food safety: barrier materials, antimicrobials and sensors," Journal of Colloid and Interface Science, vol. 363, no. 1, pp. 1-24, 2011.

[13] X. Jiang, D. Valdeperez, M. Nazarenus et al., "Future perspectives towards the use of nanomaterials for smart food packaging and quality control," Particle \& Particle Systems Characterization, vol. 32, no. 4, pp. 408-416, 2015.

[14] N. Mohseni Kiasari, S. Soltanian, B. Gholamkhass, and P. Servati, "Room temperature ultra-sensitive resistive humidity sensor based on single zinc oxide nanowire," Sensors and Actuators A: Physical, vol. 182, pp. 101-105, 2012.

[15] H. M. C. de Azeredo, "Nanocomposites for food packaging applications," Food Research International, vol. 42, no. 9, pp. 1240-1253, 2009.

[16] W. Chalco-Sandoval, M. J. Fabra, A. López-Rubio, and J. M. Lagaron, "Use of phase change materials to develop electrospun coatings of interest in food packaging applications," Journal of Food Engineering, vol. 192, pp. 122-128, 2017.

[17] O. Sarapulova, V. Sherstiuk, and V. Shvalagin, "Luminescent nanosized composites for indicating and preventing compositional changes of packaged products in modern printed packaging," Nanoscience and Nanotechnology Letters, vol. 5, no. 11, pp. 1141-1146, 2013.

[18] V. Shvalagin, G. Grodziuk, O. Sarapulova, M. Kurmach, V. Granchak, and V. Sherstiuk, "Influence of nanosized silicon oxide on the luminescent properties of $\mathrm{ZnO}$ nanoparticles," Journal of Nanotechnology, vol. 2016, Article ID 2708638, 7 pages, 2016.

[19] A. M. Díez-Pascual and A. L. Díez-Vicente, "ZnO-reinforced poly (3-hydroxybutyrate-co-3-hydroxyvalerate) bionanocomposites with antimicrobial function for food packaging," ACS Applied Materials \& Interfaces, vol. 6, no. 12, pp. 9822-9834, 2014.

[20] M. M. Berekaa, "Nanotechnology in food industry; advances in food processing, packaging and food safety," International Journal of Current Microbiology and Applied Sciences, vol. 4, no. 5, pp. 345-357, 2015.

[21] O. Hrytsenko, D. Hrytsenko, V. Shvalagin, G. Grodziuk, and $\mathrm{N}$. Andriushyna, "The influence of parameters of ink-jet printing on photoluminescence properties of nanophotonic labels based on Ag nanoparticles for smart packaging," Journal of Nanomaterials, vol. 2017, Article ID 3485968, 9 pages, 2017.

[22] M. Jung, J. Kim, H. Koo, W. Lee, V. Subramanian, and G. Cho, "Roll-to-roll gravure with nanomaterials for printing smart packaging," Journal of Nanoscience and Nanotechnology, vol. 14, no. 2, pp. 1303-1317, 2014.

[23] O. Hrytsenko, V. Shvalagin, G. Grodziuk, and V. Granchak, "Influence of parameters of screen printing on photoluminescence properties of nanophotonic labels for smart packaging," Journal of Nanotechnology, vol. 2017, Article ID 7125682, 12 pages, 2017.

[24] D. Qu, M. Zheng, P. Du et al., "Highly luminescent S, N codoped graphene quantum dots with broad visible absorption bands for visible light photocatalysts," Nanoscale, vol. 5, no. 24, pp. 12272-12277, 2013.

[25] W. U. Khan, D. Wang, W. Zhang et al., "High quantum yield green-emitting carbon dots for Fe (III) detection, biocompatible fluorescent ink and cellular imaging," Scientific Reports, vol. 7, no. 1, p. 14866, 2017.

[26] Z. Luo, Y. Lu, L. A. Somers, and A. T. C. Johnson, "High yield preparation of macroscopic graphene oxide membranes," Journal of the American Chemical Society, vol. 131, no. 3, pp. 898-899, 2009. 


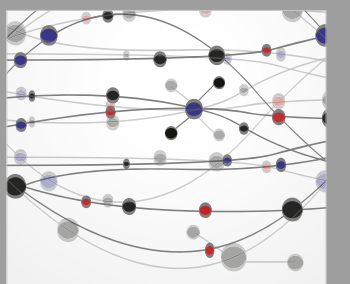

The Scientific World Journal
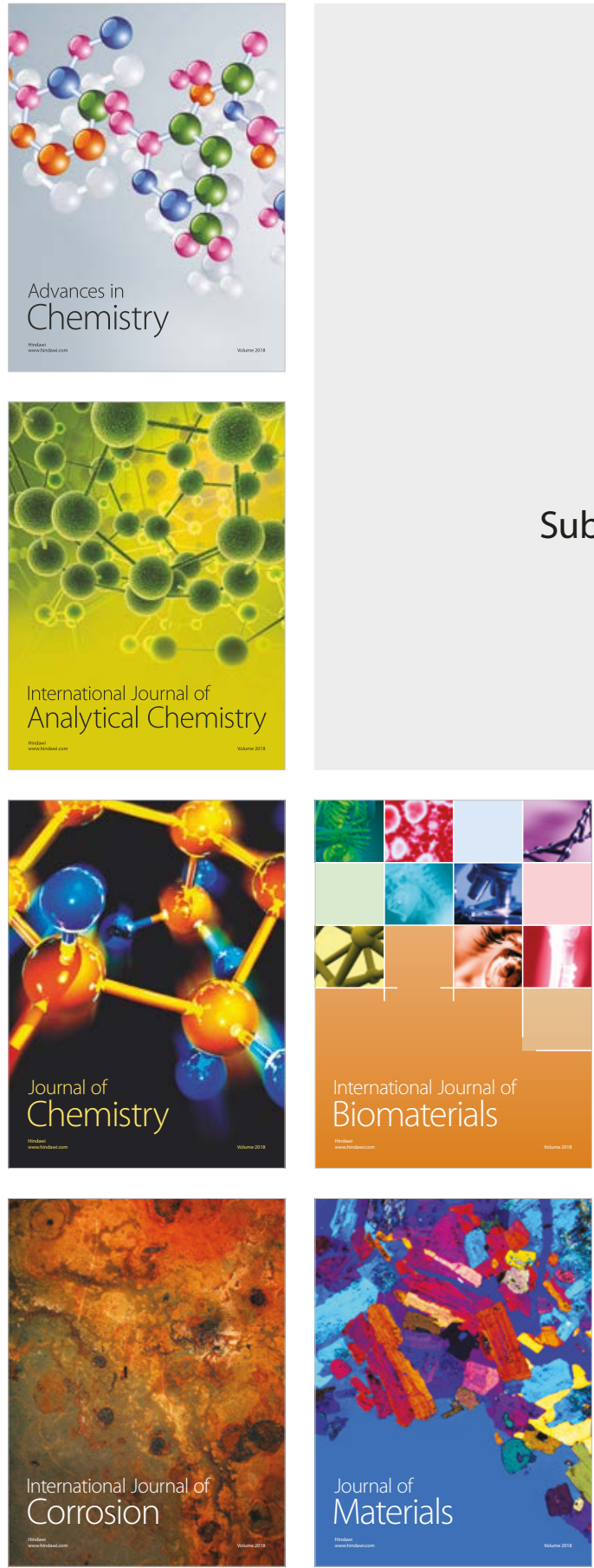

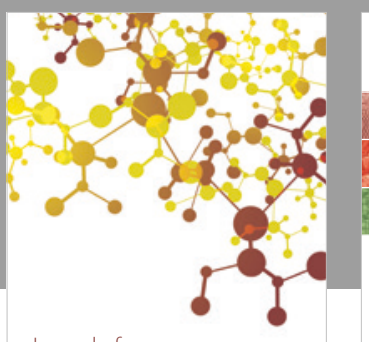

Journal of

Applied Chemistry
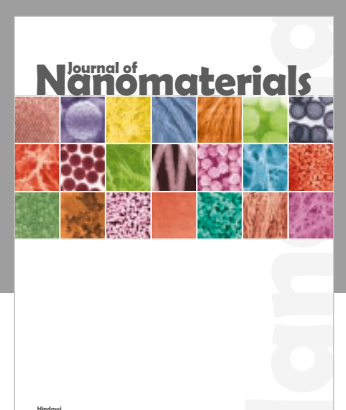

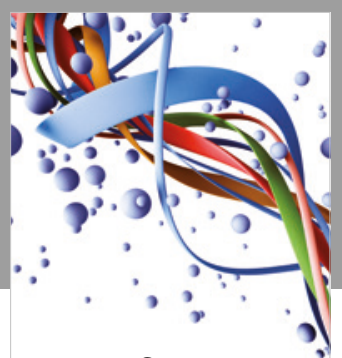

Scientifica

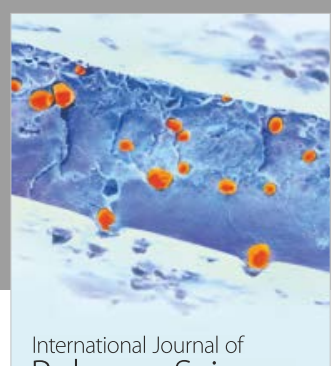

Polymer Science

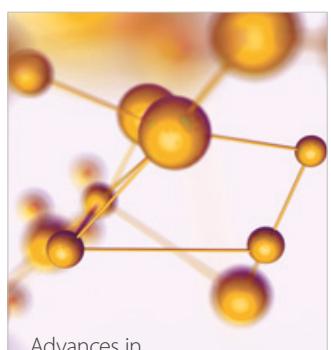

Physical Chemistry
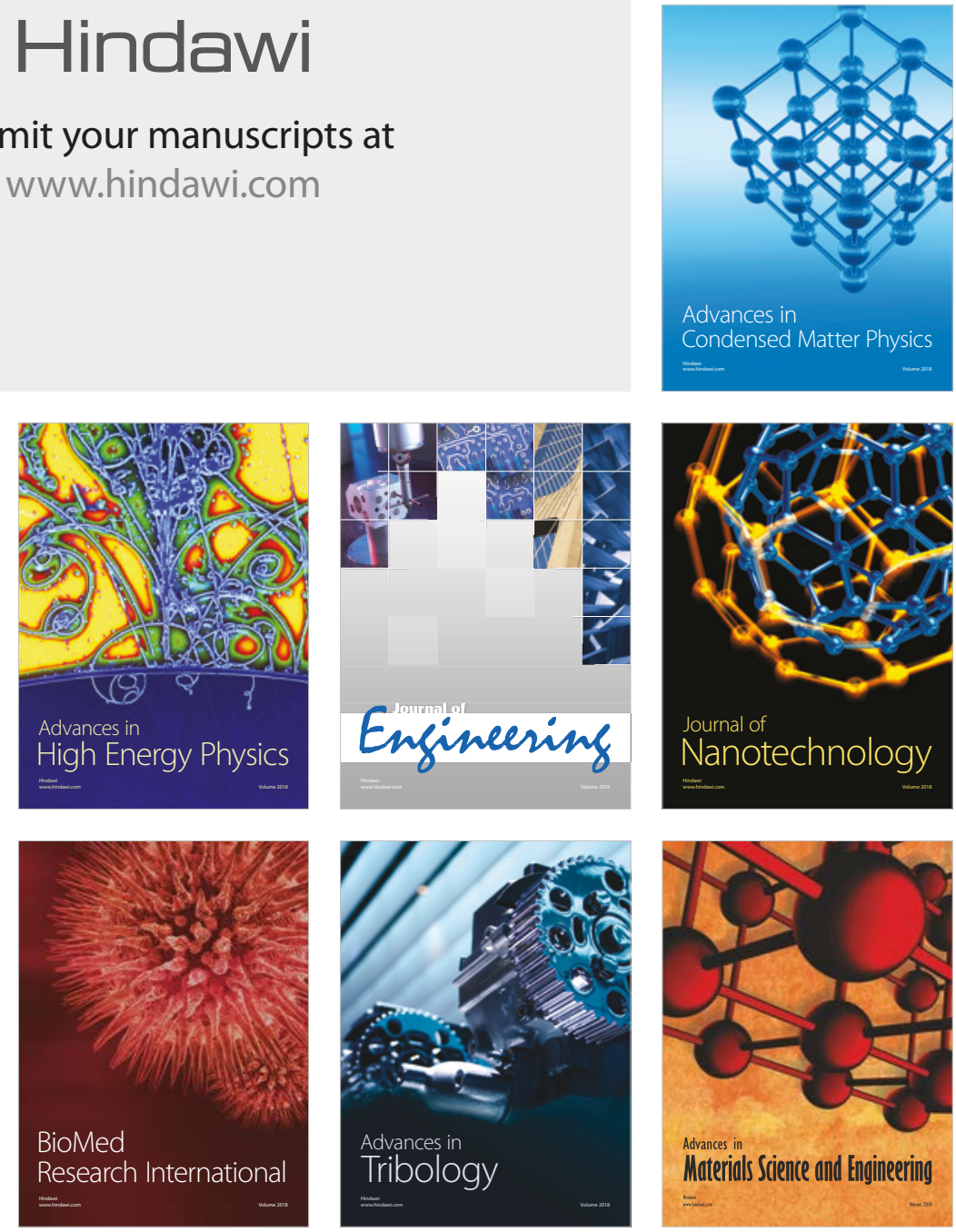